EDITORIAL

\title{
Is informed consent possible in acute myocardial infarction?
}

\author{
B A Foëx
}

Heart 2004;90:1237-1238. doi: 10.1136/hrt.2003.020255

\section{Are patients in acute myocardial infarction sufficiently competent to understand what they are being told?}

$T$ he study by Ågård and colleagues, ${ }^{1}$ published in a previous issue of Heart, raises the question of whether or not patients with acute myocardial infarction (AMI) can give informed consent to their treatment. At its most simple, "Consent is a state of mind: a decision by a patient". ${ }^{2}$ In practice consent is valid if: (1) the patient is competent; (2) the patient is informed; and (3) consent is freely given. It has been defined as: "an autonomous authorisation by individuals of a medical intervention or of involvement in research... a person must do more than express agreement or comply with a proposal."'3

Ågård and colleagues ${ }^{1}$ reported on a survey of Swedish cardiologists and their perception of the informed consent process in interventional trials in AMI. ${ }^{1}$ The vast majority $(86 \%)$ felt that patients were unable to understand all the information given to them, and so, by definition, were unable to give fully informed consent. A quarter of those who responded to the questionnaire went further. They believed that patients in AMI could never have enough understanding to give fully informed consent.

\section{UNDERSTANDING THE ISSUES SURROUNDING CONSENT}

What evidence is there that patients themselves fail to understand all the issues in the consent process, when acutely ill? Yuval and colleagues ${ }^{4}$ found that only $31 \%$ of 129 patients, who took part in the ISIS-4 trial, perceived that they had full comprehension of the trial, while $19 \%$ felt that they had no understanding of the trial. ${ }^{4}$ Poor understanding of research protocols was also reported in patients with unstable angina and non-Q wave AMI. ${ }^{5}$ The situation is no better in patients admitted with subarachnoid haemorrhage: only one third spontaneously recalled participating in a clinical trial, and only $19 \%$ of those who had given consent themselves could remember the consent procedure. ${ }^{6}$ More significantly, perhaps, was the fact that only $28 \%$ of the patients felt that they had been capable of making an adequate decision. Most recently, Williams and colleagues" found that "few patients gave consent that was truly autonomous and informed" in the Hirulog and early reperfusion or occlusion (HERO)- 2 trial. $^{7}$

Box 1 lists the possible obstacles to informed consent in the setting of AMI.

Kucia and Horowitz ${ }^{5}$ found that the presence of anxiety, dyspnoea, the seniority of the person obtaining consent, the age of the patient, and whether English was the first language of the patient did not seem to affect whether a patient would understand the information presented. Significant determinants of understanding were: (1) absence of pain at trial entry; (2) level of education; and (3) male sex. The use of morphine did not adversely affect comprehension of verbal information given in the HERO-2 study. ${ }^{7}$

It might also be thought that patients suffering AMI are too ill to give informed consent. Ågård and colleagues, ${ }^{8}$ in their 2001 study, found that "Most interviewees felt that they either had too low a level of consciousness to understand the information given, or were in too much pain to bother". Certainly Schaeffer and colleagues ${ }^{9}$ found that severely ill patients in phase 1 studies retained the least information about risks and side effects, compared to healthy volunteers and less ill patients. However this group had good retention of information about procedures. The authors suggested that patients with different disease severities engaged in different ways with the information provided about clinical trials.

In contrast to this, Yuval and colleagues ${ }^{10}$ found that the level of understanding of trial information was almost the same for patients entered into either AMI trials or outpatient heart failure trials. In both trial types about 30\% felt they had full understanding of the information and $20 \%$ felt they had little or no understanding of the trial. These findings may reflect a deficiency in the way information was presented rather than an inability to understand by the patient. This was also the conclusion of a study of participants in a non-acute gynaecological clinical trial. ${ }^{11}$

\section{WHAT INFORMATION DO PATIENTS NEED?}

As a clinician I know very well that if I inform a patient of all the risks of a procedure first then consent is more likely to be refused. The way in which information was presented was certainly a factor in the HERO-2 study. Williams and colleagues ${ }^{7}$ found that "the patient information sheet needed a higher educational level than most patients had achieved". ${ }^{7}$ The result was that only $18 \%$ of patients read the information sheet before giving or refusing consent. In the case of patients with subarachnoid haemorrhage only $9 \%$ actually read the information provided. ${ }^{6}$

There appears to be a preference for oral rather than written information. ${ }^{4}$ But what information? The debate, until recently, has centred on what information the doctor needed to disclose to avoid a claim of negligence: the "professional practice" standard. This is reviewed by Mason 


\section{Box 1: Obstacles to informed consent}

- Lack of competence, secondary to:

- pain

- opiates/drugs

- reduced level of consciousness

- impaired cerebral perfusion secondary to hypotension

- anxiety

- Failure of disclosure process, because of:

- poor communication by the doctor

- educational mismatch between patient and information provided

and McCall Smith. ${ }^{12}$ This is now being replaced by the "reasonable patient" standard, already in use in the USA. The doctor should inform the patient of any risk, which would affect the decision of a "reasonable patient" (for example, Pearce v United Bristol Healthcare NHS Trust ${ }^{13}$ ).

When it comes to disclosure time is very important. Yuval and colleagues ${ }^{4}$ found that comprehension was related to a recollected explanation of five minutes or more and to an opportunity for discussion at the time of consent. Ågård and colleagues $^{8}$ recorded that some patients felt they had too little time to decide whether or not to take part in the trials. Williams and colleagues ${ }^{7}$ found that those patients who declined to take part in the HERO-2 study were more likely to feel that they were not given enough time to consider their decision than those who decided to take part.

\section{IS INFORMED CONSENT POSSIBLE IN AMI?}

Informed consent is a prerequisite to good clinical practice, ${ }^{14}$ with some possible exceptions ${ }^{15}$ (see box 2 ).

Doyal has suggested: "The real enemy of proper informed consent in medicine is not the inability of the adult patient to engage in the process. Rather it is insufficient resources to train clinicians to communicate more successfully with their patients and inadequate staff to allow enough available time for this essential communication to occur." ${ }^{14}$ From the evidence presented this is probably not so for the majority of patients in AMI. Many of them are just too ill to give fully informed consent.

Does this mean that they cannot engage in the decision making process? No, "Consent is a state of mind: a decision by a patient". ${ }^{2}$ A patient may make a decision without very detailed knowledge, but with trust in the physician. After all, as Beauchamp and Childress ${ }^{3}$ have stated, "From the fact that actions are never fully informed, voluntary, or autonomous, it does not follow that they are never adequately informed, voluntary, or autonomous".

In a recent paper Corrigan and Williams-Jones ${ }^{16}$ Complained: "There is growing concern that ethics is being seen only as bureaucracy... Legislation must promote ethics as culture, not ethics as bureaucracy." Although AMI is a
Box 2: Exceptional circumstances, when

informed consent may not be possible/ necessary

- Public health emergency

- Medical emergency

- Incompetence

- Patient waiver

- Therapeutic privilege

medical emergency and there is good evidence that many patients are "incompetent" to make a decision, the studies cited above suggest that some patients are competent, and regard themselves as competent, to make a decision. A dialogue between the patient and physician is essential. This needs to be pitched at a level suitable for that patient, taking into account his or her clinical condition, such that he or she can make a decision. Ethicists and patient advocates must understand the constraints of delivering "time critical" treatment to the acutely ill, and clinicians must learn to empower their patients to make autonomous decisions, when they can. These are the real challenges for "informed consent" in acute myocardial infarction.

\section{REFERENCES}

1 Ågård A, Herlitz J, Hermerén G. Obtaining informed consent from patients in the early phase of acute myocardial infarction: physicians' experiences and attitudes. Heart 2004;90:208-10.

2 Aitkenhead AR. Consent and adult patients in anaesthesia and intensive care. In: Draper H, Scott W, eds. Ethics in anaesthesia and intensive care. Edinburgh: Butterworth-Heinemann, 2003:17-34.

3 Beauchamp T, Childress J. Principles of biomedical ethics, 4th ed. Oxford: Oxford University Press, 1994.

4 Yuval R, Halon DA, Merdler A, et al. Patient comprehension and reaction to participating in a double-blind randomized clinical trial (ISIS-4) in acute myocardial infarction. Arch Intern Med 2000;160:1142-6.

5 Kucia A, Horowitz J. Is informed consent to clinical trials an "upside selective" process in acute coronary syndromes? Am Heart J 2000;140:94-7.

6 Schats R, Brilstra EH, Rinkel GJ, et al. Informed consent in trials for neurological emergencies: the example of subarachnoid haemorrhage. J Neurol Neurosurg Psychiatry 2003;74:988-91.

7 Williams BF, French JK, White HD. Informed consent during the clinical emergency acute myocardial infarction (HERO-2 consent substudy): a prospective observational study. Lancet 2003;361:918-22.

8 Ågård A, Hermerén G, Herlitz J. Patients' experiences of intervention trials on the treatment of myocardial infarction: is it time to adjust the informed consent procedure to the patient's capacity? Heart 2001;86:632-7.

9 Schaeffer MH, Krantz DS, Wichman A, et al. The impact of disease severity on the informed consent process in clinical research. Am J Med 1996; 100:261-8

10 Yuval R, Halon DA, Flugelman MY, et al. Perceived patient comprehension in acute and chronic cardiovascular clinical trials. Cardiology 2003;99:68-71.

11 Lynoe N, Sandlund M, Dahlqvist G, et al. Informed consent: study of quality of information given to participants in a clinical trial. BMJ 1991;303:610-13.

12 Mason J, McCall Smith R, Laurie G. Law and medical ethics, 5th ed. London: Butterworths, 1999:277-88.

13 Pearce v United Bristol Healthcare NHS Trust [1998] 48 BMLR 118, 1998.

14 Doyal L. Good clinical practice and informed consent are inseparable. Heart 2002;87:103-6.

15 Beauchamp T. Informed consent. In: Veatch R, ed. Medical ethics, 2nd ed. Sudbury, Massachusetts: Jones and Bartlett Publishers, 1997: 185-208.

16 Corrigan OP, Williams-Jones B. Consent is not enough-putting incompetent patients first in clinical trials. Lancet 2003;361:2096-7. 